Manfred O. Hinz

\title{
Die Bestürzung, angeschaut zu werden. Deutsches im afrikanischen Visier
}

\section{Europäische Imagination and afrikanische Herausforderung}

»Der Weiße hat dreitausend Jahre das Privileg genossen anzuschauen, ohne angeschaut zu werden «, schrieb Jean-Paul Sartre in seinem 1948 erschienenen Vorwort Orphée Noir zur Anthologie de la nonvelle poésie nègre et malgache de la langue française. ${ }^{1}$ "Hier stehen Menschen vor uns, die uns anschauen, und ich wünsche euch, dass auch ihr wie ich mit Bestürzung fühlt, wie es ist, wenn man angeschaut wird. $\ll^{2}$ Mit anderen Worten: Die Zeit der in selbstkritischer Absicht unternommenen Imaginationen, für die Montesquieus Lettres Persannes exemplarisch stehen und die unter anderem in der Forschungsreise ins innerste Deutschland des erfundenen Afrikaners Lukanga Mukara eine afrikanische Nachahmung fanden, ${ }^{3}$ ist vorbei. ${ }^{4}$ Vorbei ist die Zeit der gebildeten Perser, die ein Montesquieu erfinden konnte, um unsere Welt in kritische Distanz zu bringen; vorbei die Zeit, in der Mukaras Autor Hans Paasche exotische Kulturkritik zur Propagierung eines einfachen und gesunden Lebenswandels in Deutschland üben lassen konnte. ${ }^{5}$ Vorbei ist aber auch die Zeit der Forschungsreisenden und Wissenschaftler der ethnologischen Gründerzeit aus Deutschland und anderswo, die Afrika and anderen Teilen der Welt in europäischer Vogelperspektive entgegentraten.

1 5. Aufl., Paris 1985, S. IX (deutsche Fassung zitiert nach J. Riesz (Hg.), Blick in den schwarzen Spiegel. Das Bild des Weißen in der afrikanischen Literatur des 20. Jahrbunderts. Wuppertal 2003, S. 11.

2 Ebd.

3 H. Paasche, »Die Forschungsreise des Afrikaners Lukanga Mukara ins innerste Deutschland «; zitiert nach dem Auszug in: G. Stein (Hg.), Exoten durchschanen Europa. Der Blick des Fremden als ein Stilmittel abendländischer Kulturkritik. Von den Persischen Briefen im 18. bis zu den Papalagi-Reden des Südseehäuptlings Tuiavii im 20. Jabrbundert. Ethnoliterarische Lesebücher. Bd. 2. Frankfurt/M. 1984, S. $216 f f$.

4 Auch wenn, wie H. Behrend (Einleitung zu: H. Behrend /T. Geider (Hg.), Afrikaner schreiben zurück. Texte und Bilder afrikanischer Ethnographen. Köln 1968, S. 21) zurecht vermerkt, dass die verkehrten Ethnographien des 17. und 18. Jahrhunderst »bis heute ihre Faszination nicht verloren « haben, ist die Faszination heute anders als damals mehr durch Sprache und Stil der Texte begründet als durch exotische Neugierde. Vgl. hierzu, was P. Schunck (Nachwort. In: P. Schunck (Hg.), Montesquien Persische Briefe. Stuttgart 1991, S. 355ff.) zur Wirkung der Briefe nach ihrem Erscheinen zu sagen hat.

5 Siehe hier Steins Vorwort in: aaO. (FN 3), S. $12 \mathrm{f}$. 
Der Afrikaner Yambo Ouologuem karikiert die mangelnde Augenhöhe deutscher Ethnologie in seinem Roman Le devoir de violence, ${ }^{6}$ indem er aus dem Begründer der Frankfurter kulturhistorischen Schule Leo Frobenius Fritz Schrobenius werden lässt: Ouloguem beschreibt Schrobenius als einen großen, füllig stattlichen Deutschen mit roten Bartkoteletten, blühender Gesichtsfarbe, blauen, zärtlichen und ernsthaften Augen und einem schon sichtbaren Bauch.' Mit Schrobenius Ethnologie hat Ouloguem Probleme: »... dieser menschliche Krebs träumte nämlich davon, unter der Flagge der kulturellen Autonomie eine afrikanische Renaissance herbeizuführen, die allerdings zur lebendigen Gegenwart keinerlei Beziehung mehr hatte. $\ll^{8}$ Schrobenius ist nicht nur der Prototyp eines, der anschaut, ohne sich anschauen zu lassen; er ist der Prototyp des Ethnologen, der offensichtlich selektiv, d. h. ohne Versuch zur Distanz nach Maßgabe seines mitgebrachten Verständnisses wahrnimmt. Dabei übersieht Ouologuem nicht, dass der eingeschränkte Blick von Schrobenius auf afrikanischer Seite Verhalten produziert, das wiederum der eingeschränkten Wahrnehmung Schrobenius entgegenkommt. Ouloguem kommentiert diese Dialektik in einer genussvoll üppigen Sprache. Nachdem Schrobenius die mitgebrachten Geschenke ausgebeitet hatte und diese im Palast des von Schrobenius besuchten Herrschers verteilt waren, küsste die »kniende Menge... in einem Delirium der Dankbarkeit die Erde - die Gebete flossen ihr von den Lippen in einer ziternden Litanei ersterbender Worte, die sich, wie die Flamme eines fast erloschenen Brandes, zeitweise neu belebte. « ${ }^{9}$ Auch die Informanten von Schrobenius regaieren auf den Forscher. Der Informant fabuliert und der Übersetzer überspitzt die Feinbeiten, an denen sich der Forscher begeistert. ${ }^{10}$

Mit seiner Kritik an der von Schrobenius betriebenen Ethnogie hat Ouloguem eine spezifische Variante der in Deutschland betriebenen Wissenschaft Ethnologie in den Blick genommen, - die romantizierende kulturhistorische Suche, die nach den Bildern großer Vergangenheit in der nur Bruchstücke transportierenden Gegenwart sucht. Insoweit geriet ihm Deutsches ins Visier. Die Kritik der Methode allerdings hat jenseits der deutschen Grenzen Bedeutung. Was hier im Visier ist, ist Problem der Ethnologie wo immer und von wem auch immer sie betrieben wird, wobei die Nach-Schrobenius Ethnologie aus Kritik gelernt hat. Ein deutsches Bild gelingt Ouologuem dann dort wieder, wo er die Erscheinung von Schrobenius beschreibt: Es entsteht das Bild eines fast typisch Deutschen, auch wenn die Beschreibung, stünde sie in einem anderen Kontext, mit geringer Anpassung zu der eines Holländers, Dänen oder sonstigen Nordeuropäer werden könnte.

Für die Suche nach Deutschem im afrikanischen Visier ist Ouloguems Roman ein Glücksfall. Ein Glücksfall, weil (der reale) Frobenius und seine Exkursionen nach Westafrika Spuren in der Wahrnehmung von Afrikanern hinterlassen haben, die bis

6 Paris 1968.

7 Vgl. den deutschen Auszug aus Ouloguems Roman bei: Riesz, aaO. (FN 1), S. 209.

8 Ebd.

9 Ebd.

10 Ebd. 
heute provozieren. ${ }^{11}$ Wo diese bearbeitet werden (hier oder in Afrika), wird zwangsläufig auch Deutsches bearbeitet. Im übrigen geht es dort, wo der afrikanische Blick den Augenkontakt zum vormalig alleinigen Beschauer aus Europa sucht, nur ausnahmsweise spezifisch um einen Blick auf den Deutschen und das Deutsche. Der Blick richtet sich in erster Linie auf den kolonialen und post-kolonialen Weißen, wobei in Europa mehr oder weniger gängige und weitverbreitete Bilder zum Unterschied von Angehörigen der verschiedenen europäischen Nationen geringe Bedeutung haben. Was sich der Wahrnehmung der Innenschau oder der Schau des Nachbarn offensichtlich einfach und fast selbstverständlich anbietet, verliert die Konturen in der Ferne. ${ }^{12}$

Die folgenden Überlegungen werden diese These aufgreifen. Im ersten der folgenden drei Abschnitte wird es um die Wahrnehmung des kolonialen Weißen im Medium afrikanischer Holzsarbeiten, vielfach als Colonfiguren bezeichnet, gehen, in denen Erscheinungsformen der weißen Kolonialisten in einer aus europäischer Sicht überstilisierten Weise verarbeitet werden. ${ }^{13}$ Colon ist die im Französischen in verächtlichmachender Absicht gebrauchte Bezeichnung für den weißen Siedler. Die Anmerkungen zur Colonkultur leiten zu einem von dieser Kultur scheinbar weit entfernten, aktuellem Thema über: der postkonialen Wahrnehmung von kolonial Deutschem, dem Hererokrieg in der ehemaligen Kolonie Deutsch-Südwestafrika und seiner Verabeitung in einem jährlich in Namibia begangenen Gedenktag.

Im anschließenden Abschnitt wird auf den in deutscher Wissenschaft und deutscher Presse vielbeachteten Beitrag eines afrikanischen Ethnologen aus jüngerer Zeit eingegangen werden, in dem dieser die die sozio-kulturelle Bedeutung von Hunden in Deutschland als Zugang zum Verständnis des Deutschen wählte. Im dritten und letzten Teil soll einiges zur Wahrnehmung des Deutschen aus dem Alltag eines afrikanischen Landes Berichtet werden, indem Deutsches auf Grund der Rolle, die Deutsche spielen, auf ungewöhnliche Weise sichtbar ist: Namibia.

\section{Colonfiguren - The savage hits back - und der Hererotag in Okahandja}

The savage hits back ist der Titel eines im Jahre 1937 erschienen Buches von Julius Lips. ${ }^{14}$ Lips Anliegen war es, sogenannter primitiver Kunst ${ }^{15}$ einen eigenständigen Platz in der Kunstbetrachtung einzuräumen, oder anders, die Gestaltungen au-

11 So hat Léopold Sédar Senghor in seinen Arbeiten zur Négritude unter anderem auch Frobenius rezipiert.

12 Dies gilt etwa für das Kompendium deutscher Urworte, besser Urkonzepte, wie sie H.D. Gelfert (Was ist deutsch? Wie die Deutschen wurden, was sie sind. München 2005, S. 22ff.) erörtert.

13 Obwohl die hohe Zeit der Colonkultur im ersten Teil des vorigen Jahrhunderts anzusetzen ist, gibt es Colonwerke bis heute. Aus Beobachtungen des Autors im südlichen Afrika (Angebote in Kunsthandlungen, Dekorationen in Hotels) könnte man sogar von einer Renaissance der Colonkultur sprechen, auch wenn das, was den Markt bestimmt, von eher schlicher Formgebung ist. 
Bereuropäischer Völker in ihrer Eigenwertigkeit zu erfassen. Besonderes Augenmerk richtet Lips dabei auf Erscheinungen in dieser Kunst, in denen Elemente aus dem Kontakt mit dem weißen Kolonialherren eingearbeitet werden. Eine im Jahre 1983 im Münchener Stadtmuseum gezeigte Ausstellung Colon. Das schwarze Bild vom weißen Mann enthielt unter anderem zahlreiche Arbeiten aus Togo, einer der ehemaligen afrianischen Kolonien Deutschlands. ${ }^{16}$ Fast alle männlichen Figuren haben typische, aus dem europäischen Kontext kommende und zum Teil in ihrer Ausgestaltung überbetonte Kopfbedeckungen, Helme, Militärmützen, Hüte. Selbst ein im übrigen nackter Mann trägt eine Art Militärmütze. Oftmals umgreift die ansonsten nicht tailierte Mitte der Figuren ein massiver, breiter Gürtel, an dem Revolvertaschen hängen, oder auch Säbel. Markante, senkrecht ausgestellte kurze Hosen gehen in stramme Beine mit Kniestrümpfen über. An den Oberkörpern fallen an vielen Figuren übergroße Hemdtaschen auf.

Lips zentrale These im Sinne des englischen Titels seines Buches ist, dass Verarbeitungen weißer Elemente in Darstellungen von Menschen mit mehrheitlich schwarzen Merkmalen Ausdruck von Widerstand ist, Widerstand durch Lustig-machen, durch karikierende Überbetonung der als besonders, d. h. als anders empfundenen Attribute der Weißen. »Verkennen wir es nicht« sagt Lips, »jenes erste Lächeln über den weißen Mann bedeutete auch ein erstes Aufbäumen. Die seltsamen Abbilder der Fremden aus Europa und Amerika zeigen uns..., dass der Farbige die Welt der Weißen erkannt hat, dass er um ihre Schwächen und Fehler weiß.... «17

Auseinandersetzungen mit der Colonkultur, die auf Lips folgten, haben zu wichtigen Modifikationen der These von Lips geführt; Modifikationen, die in seiner Arbeit, schaut man genauer hin, bereits angelegt sind. Im Abschnitt Seltsame Dinge um den weißen Mann berichtet Lips eine Anekdote, in der ein alter Kameruner einen Missionar um dessen Hut bittet - man errinnere, dass fast alle der in der Münchener Ausstellung zu sehenden Figuren aus Togo mit eine Kopfbedeckung tragen: »Wenn ich tot bin«, erklärte der Bittsteller, »setze mir deinen Hut auf, dann denken die im Totenreich, es kommt ein Europäer « ${ }^{18}$ Obwohl der alte Man offensichtlich kein christlich geprägtes Verständnis vom Leben nach dem Tod hatte, setzte er dennoch auf ein weißes Element, um sich einen Zugangsvorteil für das Totenreich zu verschaffen, den Hut des Missionars. Vielleicht war die Bitte an den Missionar von einem leicht spöttischen Lächeln begleitet, mit dem die Ernsthaftigkeit der Bitte ein

14 2. Ausgabe. New York 1966. Die deutsche Ausgabe des Buches (Der Weiße im Spiegel der Farbigen. Leipzig - für die Bundesrepublik Deutschland München 1983) wurde von Eva Lips besorgt. Julius Lips, Professor für Ethnologie an der Universität Köln, emigrierte 1933 nach den USA und starb nach seiner Rückkehr als Rektor der Universität Leipzig im Jahre 1950.

15 Sogenannter primitiver Kunst allgemein, d. h. nicht nur Kunst aus Afrika.

16 J. Jens (Hg.), Colon. Das schwarze Bild vom weißen Mann. Eine Ausstellung im Münchener Stadtmuseum 18. Februar bis 17. April 1983. München. 1983; siehe besonders nach S. 64, Abbildungen 65-104.

17 Ebd., S. 66.

18 Ebd., S. 147. 
Stück weit relativiert wurde: Sollte der Hut den Zugang zum Totenreich erleichtern: gut! Würde er nicht helfen, so würde er auf jeden Fall auch keinen Schaden bringen. In diesem Sinne ist die Colonkultur mehr als Verhöhnung der verhassten Weißen; sie ist auch Verarbeitung von Fremdem in das eigene Weltbild. Hierin liegt ihre außergewöhnliche Kreativität. Die Darstellungen weißer Autoritäts- und Machtsymbole sind Versuche diese im Kontext der eigenen Kultur zu begreifen und handhabbar zu machen. Die Colonkultur dient, wie Fritz Kramer sagt, der Arbeit am Trauma des Kolonisierten. ${ }^{19}$

Arbeit am Trauma des Kolonisierten ist auch, was in Okahandja, einer mittelgroßen Stadt und eine Autostunde von der Hauptstadt Namibias, Windhoek, entfernt, alljährlich im August am sogenannten Hererotag geschieht:

Die Herero waren (neben den im südlichen Teil der ehemaligen Kolonie Deutsch-Südwestafrika lebenden Nama) die Bevölkerungsgruppe, die am meisten unter dem deutschen Kolonialismus zu leiden hatte. Sie lebten in einem Gebiet, an dem die weißen Siedler in besonderer Weise interessiert waren. ${ }^{20}$ Das schwierige Verhältnis zwischen deutscher Kolonialmacht und Herero kulminierte im Jahre 1904. Es kam zum Krieg zwischen Deutschland und den Herero. Im August 1904 fand die entscheidende Schlacht von Hamakari (Waterberg) statt, mit der der Widerstand der Herero brach. Im Oktober des gleichen Jahres gab der deutsche Befehlshaber, General von Trotha, den berühmt-berüchtigten Vernichtungsbefehl bekannt, nachdem jeder Herero »mit oder ohne Gewehr, mit oder ohne Vieh« zu erschießen sei.

Die Schlacht von Hamakari bedeutete das Ende des Krieges. Die Herero flohen, wobei sie verschiedene Fluchtwege nutzten. Ein Teil erreichte Botswana, tausende starben. Angehörige der deutschen Truppe berichten vom Leid der Menschen und Tiere im Sandveld, dem Gebiet zwischen Waterberg und Botswana. Für diejenigen, denen Flucht nicht gelang, folgten Lager, Zwangsarbeit und Enteignung. Die Enteignungen von Land und Vieh sind zusammen mit dem späteren Verbot der Großviehzucht in ihrer Auswirkung auf die soziale und politische Ordnung der Herero nicht zu unterschätzen. Die Wegnahme von Land ist weit mehr als Eingriff in vermögenswerte Rechte. Wegnahme von Land ist Verlust der politisch-ökonomischen Grundlage, Enteignung des Viehs und Viehzuchtverbot - die Rinder verbinden mit den Ahnen - bedeuten Verlust der geistig-spirituellen Identität der Gemeinschaft; Tatbestände, die von der überwiegenden Meinung in Wissenschaft und Politik als Völkermord gewertet werden. ${ }^{21}$

Die Hererotage stehen im Zeichen dieser Geschehnisse. Herero kommen an diesen Tagen aus allen Teilen des Landes, selbst aus dem benachbarten Botswana, in-

19 F. Kramer, »Die Fremdheit afrikanischer Colon-Figuren« in: J. Jens, aaO. (FN 16), S. 210.

20 Hierzu und zum folgende: M O Hinz, »Der Krieg gegen die Herero: Friedensschluss hundert Jahre danach? « in: N. Paech / A. Rinken / D. Schefhold / E. Wesslau, (Hg.), Völkerrecht statt Machtpolitik. Beiträge für Gerhard Stuby. Hamburg 2004, $148 \mathrm{ff}$.

21 Auch hierzu finden sich im gerade zitierten Beitrag von Hinz, aaO. (FN 20) Hinweise. 
dem immer noch Nachkömmlinge der 1904 aus Namibia geflohenen Herero leben. Neben den rituellen Besuchen an den Hererogräbern wie aber auch öffentlichen Kundgebungen finden Paraden und Märsche der Hererotruppen (in der Sprache der Herero Otrupe genannt) statt. Wer sich als Herero versteht, gehört der Otrupe an. Die Teilnehmer der Otrupe-Paraden tragen an die deutsche Schutztruppe erinnernde Uniformen, wobei durchaus auch Bestandteile anderer Truppenbekleidungen hinzugefügt werden (wie zum Besipiel Orden der DDR Volksarmee, wie der Autor dieses Beitrages vor Ort sehen konnte). Marschiert wird nach deutsch klingenden Befehlen. Otrupe ist nach deutschen Rängen gegliedert; die Befehlshaber der Otrupe tragen deutsche Namen.

Ethnohistorische Untersuchungen legen dar, wie es zur Gründung von Otrupe kam: Otrupe entstand nach Mustern, die man beim Sieger beobachtet hatte und wurde sehr schnell für die Überlebenden des Krieges, die überall im Land zertsreut waren, Netzwerk, indem man sich fand und durch das man dem drohenden völligen Untergang der Herero Nation entgegenwirken konnte. Strukturen und Formen der Sieger wurden übernommen, weil sie die offensichtlich stärkeren Kräfte symbolisierten. $^{22}$

Diese der Selbstbehauptung dienende Teilidentifizierung mit dem ehemaligen (deutschen) Feind wirkt bis heute im Verhältnis der Herero zu den Deutschen nach. Auch wenn für die meisten Herero die Wunden des Hererokrieges von 1904 nicht geheilt sind, das Trauma des Krieges also noch immer nicht verarbeitet ist, ist zwischen Herero und Deutschen nur selten ein Gefühl von Feindschaft festzustellen. Das Verhältnis ist vielmehr eine Mischung widersprüchlicher Elemente, so widersprüchlich wie die Übernahme der Uniform des Siegers. In dieser Mischung verbinden sich Achtung, bis Sympathie, Vorsicht und Wachsamkeit. So erzählt ein Herero von einen deutschsprachigen Farmer, dem Eigentümer einer Farm im einstigen Hereroland, wie dieser zur Zeit der südafrikanischen Verwaltung und trotz Apartheid eine enge Beziehung zu Herero unterhielt. Der Farmer setzte sich sogar dafür ein, dass Mitglieder einer Hererofamilie, die ungeachtet des Verlustes ihres Landes unter anderem an diesen Farmer die Beziehung zu eben diesem Land nie aufgegeben hatte, auf seiner Farm beerdigt werden konnten, also dort zu Ruhe kommen konnten, wo schon ihre Ahnen zu Ruhe gekommen waren. ${ }^{23}$ Der Bericht endet mit dem Kommentar des Erzählers: »Er (der Farmer, MOH) war kein besonders guter Mann

22 Vgl. hierszu: W. Werner, ", Playing soldiers': The Truppenspieler movement among the Herero o Namibia, 1915 to ca. 1945 « in: Journal of Southern african Studies, 1990, 16,3, S. 485ff.; J.-B. Gewald, »Herero annual parades: Commemorating to create in: Behrend / Geider, aaO. (FN 4), S. 131ff.; G. Krüger, Kriegsbewältigung und Geschichtsbewußtsein. Realität, Deutung und Verarbeitung des deutschen Kolonialkriges in Namibia 1904 bis 1907, Göttingen 1999.

23 R. L. Zaire / H. Ptemann, »Zurück nach Voigtland und Otjituezu. Erinnerungen an Penaani Fritz Zaire« in: N. Mbumba / H. Patemann / U. Katjivena, Ein Land, eine Zukunft. Namibia auf dem Weg in die Unabhängigkeit, Osnabrück 1988, S. $318 \mathrm{ff}$. 
und kein besonders schlechter Mann. Er war bestimmt klug. «24 Diese Klugheit war es, die zählte und mit der man als Herero zu rechnen hatte!

\section{Die Deutschen und die Hunde}

»Es sind vor allem US-amerikanische FeldforscherInnen, die sich in den letzten 10 Jahren in größerer Zahl in Deutschland aufgehalten haben als in den 100 Jahren davor. Aber auch zahlreiche KulturanthroplogInnen aus Nachbarländern wie Frankreich, England, Dänemark, den Niederlanden gehen in Form von Feldstudien den neuen ,incertitudes allemandes' nach, « stellen Thomas Hauschild und Bernd Jürgen Warnecken in ihrer Einleitung Entdeckungsfabrten nach Deutschland des Aussatzbandes Inspecting Germany fest. ${ }^{25}$ Inspecting Germany fasst die Beiträge einer Tagung zum gleich Thema zusammen, die im Jahre 1999 in Tübingen stattfand. Unter anderen trug der Kameruner Ethnologe, Flavien Ndonko, promoviert in diesem Fach an der Universität Hamburg, zur Tagung bei. Der Titel seine Studie: Deutsche Hunde. Ein Beitrag zum Verstehen deutscher Menschen. ${ }^{26}$

Bevor Ndonko nach Deutschland kam, studierte er an der Universität von Yaoundé. Das Deutschlandbild, das ihm an dieser kamerunischen Universität vermittelt worden war, war das eines Landes, dessen Menschen hart arbeiten, diszipliniert, aufrichtig und effizient sind. Als Kameruner hatte er die Haltbarkeit der in der deutschen Kolonialzeit errichteten Gebäude schätzen gelernt, wie aber auch die power der von Mercedes hergestellten Fahrzeuge, wie sie vom Kameruner Jetset aber auch von Hochstaplern bevorzugt werden. (So Ndonko.) Sein Interesse für deutsche Hunde, veränderte dieses Bild.

Mit deutscher Relität konfrontiert fand Ndonko eine Fülle von Themen, die ihn als ethnologischen Beobachter Deutschlands (das Land seines Stammes von »Primitiven «) ansprachen: Neben Hunden erwog er als Forschungsfelder, die ihm den ethnologischen Blick auf Deutschland erlauben würden, das Kochen von Eiern, die Behandlung älterer Menschen, das Verhalten in Fahrzeugen des öffentliche Nah- und Fernverkehrs, die Einstellung zum Auto, Werbung, die Begeisterung für Antiquitäten und anderes mehr. Es blieb jedoch bei den Hunden, ein Thema, das er sich nach den Regeln professioneller Ethnologie erschloss: Hundeversicherungen, Schönheitssalons für Hunde, Schönheitswettbewerbe, Hundekliniken, Hundefriedhöfe, Hundenahrung, der Hund als Wirtschaftfaktor (schließlich gab es 1989 in Deutschland 38 Millionen Hunde, die Hunde im anderen noch nicht wiedervereinigten Teil Deutschlands nicht mit gezählt), Hundekörperpflege. Im Ergebnis findet Ndonko das, was

24 Ebd., S.314.

25 T. Hauschild / B. J. Warnecken (Hg.), »Entdeckungsfahrten nach Deutschland « in: Dies., Inspecting Germany. Internationale Deutschland Ethnographie der Gegenwart, Münster/Hamburg/London 2002, S. 19.

26 F. Ndonko, »Deutsche Hunde. Ein Beitrag zum Verstehen deutscher Menschen« in: Hauschild / Warnecken, aaO. (FN 25), 53ff. 
er die Vermenschlichung des Hundes nennt. ${ }^{27}$ Die Gründe dafür sieht Ndonko in der Desintegration der Familie. Dabei gilt, dass die Hunde durch »Füttern, Fürsorge und ungeteilte Aufmerksamkeit ... in einen Prozess der Humanisierung einbezogen (werden, $\mathrm{MOH})$, der die Hunde ebenso verändert wie ihre Herren. $\ll^{28}$ Neben dieses Ergebnis stellt Ndonko ein sozialpsychologisches, das über den Zusammenhang von Hund und Familie hinausweist: Anders als der Mensch lässt der Hund seinen Herren uneingeschränkt herrschen. »Ist das der tiefere Grund für die wachsende Beliebtheit der Hunde?«, fragt Ndonko. »Werden die Hunde, ob sie wollen oder nicht, zum Medium einer Strategie der Intoleranz und der Herrschaft, die es dem modernen Menschen erlaubt, in einer von rechtlichen Regulierungen und Mechanismen der Strafe nur so durchzogenen Gesellschat ihre Machtinstinkte auszuleben? «29

Die Herausgeber von Inspecting Germany bekennen, dass für sie die ungewöhnliche Aufmerksamkeit, die Ndonkos Beitrag fand, irritierend war, da »man doch alles über Hunde in Deutschland zu wissen schien, auch das Merkwürdige und manchmal Slandalöse, was deutscher Tierliebe anhaften kann. «30 Erst bei näherem Hinsehen erkannten sie, dass Ndonkos Botschaft letztlich nicht in der Entdeckung eines angeblichen Charakterzugs des Deutschen lag, nämlich zum Gehorsam eines Hundes fähig zu sein, sondern in der Betonung von etwas, was hinter der flächendeckenden Hundekultur steht - und dies nicht nur der Hundekultur Deutschlands sondern auch anderer westlicher Länder, deren Hundekultur sich von der Deutschlands nicht allzusehr unterscheidet. »Ndonko ... geht es um die pure Masse der den Hunden gewidmeten Nahrungsmittel, um die surreale Präsenz der Tiere auf Autobahnen oder in Zügen, um Hunde als vollwertige Mitglieder von Familien und Haushalten. Er sieht unser Land als Normalfall einer Überflussgesellschaft. « ${ }^{31}$ Er hält ihr, ethnologische Freiheit nutzend, seinen eigenwillig geformten Spiegel vor und erfährt Bestürzung von denen, die immer noch nicht gewohnt sind, angeschaut zu werden.

\section{Marrying-up, schwarze Deutsche, weiße Afrikaner, Deutschländer oder: die Wabrnehmung des Deutschen im Prozess}

Ndonkos Forschung provozierte bereits Erstaunen bis Unverständnis, als er auf Feldforschung in Deutschland unterwegs war: In Deutschland gab man ihm zu verstehen, er solle sich doch statt um Hunde in Deutschland um die Armut in seinem Lande kümmern, in Kamerun wollte man ihm nicht glauben, dass in Deutschland eine derartige Hundekultur existierte, und lachte über die von ihm berichteten Ergebnisse. Vermutlich steht die deutsche Kritik am Hundethema des Kameruners der Irritation, die die Herausgeber von Inspecting Germany verspürten, nicht fern. Die Reaktion in Kamerum ist auf jeden Fall normal im afrikanischen Sinne, oder, um es

27 Ebd., S. 67ff.

28 Ebd., S. 72.

29 Ebd.

30 Hauschild / Warnecken, Entdeckungsfabrten nach Deutschland, aaO. (FN 25), S. 32.

31 Ebd. 
vorsichtiger auszudrücken, üblich in dem Kontext, mit dem der Autor dieses Beitrages vertraut ist, Namibia. Man lacht, oft in leicht verschämter Weise, über Dinge, die man nicht versteht, man überlacht sie sozusagen, um zur eigenen Normalität zurückzukehren, der Normaltät eines Landes mit deutsch-kolonialer Vergangenheit und bis heute ökonomisch wichtiger und kulturell spürbarer Präsenz. ${ }^{32}$

Während das Bild des Deutschen, wie es Ndonko an seiner Universität in Kamerun erfuhr, in mehr oder weniger identischer Art in anderen Teilen Afrikas (auch wenn es dort keine deutsch-koloniale Vergangenheit gibt) zu finden sein dürfte, wird eben dieses Bild in einem Land, in dem in vielen Zusammenhängen Deutsches spürbar ist, sehr viel konkreter verstanden und entsprechend gehandhabt. Deutsche Tüchtigkeit, Ehrlichkeit und Effizienz wird gesucht und eingesetzt. Bier wird nach deutschem Reinheitsgebot gebraut und mit Nationalstolz dem aus Südafrika importierten vorgezogen. Man hat sich an den in den größeren Städten alljährlich stattfindenen Karneval nicht nur gewöhnt, ${ }^{33}$ sondern beteiligt sich als schwarzer Namibia an den Umzügen. Auch wenn man deutsch nicht wirklich spricht oder versteht, sagt man gerne Gutten Taag, jaah, jaah, um dann wieder lachend in englisch oder afrikaans weiterzureden. ${ }^{34}$ Während für die Assoziation von deutsch und Sauerkraut zur Charakterisierung des Deutschen in anderen Teilen der Welt offensichtlich ein Bedarf besteht, ist Sauerkraut, Schnitzel und anderes Teil der deutschen Spielart der namibischen Küche. Neben den Angeboten der chinesischen, italienischen, indischen und afrikanischen Küche findet man die deftigere Spielart der deutschen Küche und isst sie, aber auch die feinere und isst sie ebenfalls.

Schwarze Namibier ziehen oft, wenn sie die Wahl haben, vor, als Hausangestellte bei Weißen oder mehr noch bei Deutschen und nicht bei Schwarzn zu arbeiten. Man erwartet dort bessere Entlohnung und größere Zuverlässigkeit in der Handhabung der Arbeitsverhältnisse. Für viele junge Mädchen ist Heirat mit einem Deutschen erstrebenswerter als Heirat mit einem aus der eignen Gruppe. Obwohl es keine Statistiken hierzu gibt, spricht der Augenschein dafür, dass Heirat mit einem Deutschen besonders für Töchter aus farbigen Familien (d. h. Familien die aus Ehen zwischen Schwarzen und Weißen entstanden sind) oder Rehobother Familien (Rehoboth ist ein Gebiet im Süden Windhoeks, in dem aus dem Kap eingewanderte

32 Von den etwa 1,7 Millionen Einwohnern Nmaibias sind weniger als 20000 deutsch oder deutscher Herkunft. Deutsche sind im Geschäftsleben der größeren Städte, im kommerziellen Farmbereich und im Tourismus bestimmend. Es gibt deutsche Privatschulen, Deutschunterricht an einigen Staatsschulen, eine deutschsprachige Zeitung, ein deutsches Radioprogramm und eine deutsche lutherische Kirche. Deutschstämmige NambierInnen bekleiden Positionen auf den verschiedenen Ebenen der Regierung, bis hin zu Positionen im Ministerrang.

33 Der namibische Karneval findet sehr viel später als der deutsche statt: Es ist dann nicht mehr so heiß wie zur Zeit des deutschen Karnevals, außerdem erlaubt die Zeitverschiebung Büttenredner aus Deutschland einzuladen.

34 Afrikaans entwickelte sich aus dem Niederländischen, das die ersten Siedler ans Kap mitbrachten. Afrikaans ist heute auch Muttersprache vieler Nichtweißer in Südafrika und Namibia und weitverbreitete lingua franca. 
Nachkommen von Verbindungen zwischen Weißen und vor allem Nama leben) interssant ist. Der besondere Liebreiz der Mädchen aus Rehoboth (zumindest vom deutschen Schönheitsideal betrachtet) sorgt offensichtlich dafür, dass Deutsche gerne die Verbindung mit Rehobotherinnen suchen. Für Rehobotherinnen bedeutet Heirat mit einem Weißen (einem Deutschen) vielfach marrying-up: auf eine angesehenere soziale Ebene hinaufzuheiraten.

Die Tatsache, dass zur Zeit des Befreiungskrieges rund vierhundert schwarze Namibier und Namibierinnen in der DDR aufgewachsen waren, hat dem namibischen Bild des Deutschen eine zusätzliche Qualität verliehen. ${ }^{35}$ Bis zum Jahre 1978 hatte die namibische Befreiungsorganisation SWAPO im südangolanischen Cassinga ein Flüchtlingslager unterhalten. Bei einem Angriff der südafrikanischen Armee kamen viele der im Lager Lebenden ums Leben. Nach diesem Massaker von Cassinga boten mit SWAPO befreundete Länder, darunter die DDR, Ausbildungsplätzen für junge Namibier und Namibierinnen an. Im Unabhängigkeitsjahr 1990 kehrten sie nach Namibia zurück. Viele hatten die wichtigsten Jahre ihrer Kindheit in der DDR verbracht und waren trotz namibischen Lehrpersonals in der DDR zu schwarzen Deutschen geworden. Viele sprachen recht und schlecht die Sprache der namibischen Mehrheit oshiwambo; für die Kommunikation in der Gruppe hatte sich eine Gemisch von oshiwambo und deutsch entwickelt, das man oshidentsch taufte. $\mathrm{Zu}-$ rück in Namibia begann der schwierige Weg der Reintegration und Reidentifikation, ein Weg der tastenden Landsuche auf der Seite der Zurückgekehrten, ein Weg voll Staunen, Anteilnahme aber auch verhaltener Ablehnung auf der Seite der schwarzen Verwandten und Bekannten. Einigen half ihr Deutschsein, um sich in deutsch-geprägte Zusammenhänge einzufügen, etwa in die Tourismusindustrie, wo deutsche Kompetenz gefragt ist. Andere waren in der Lage, eine Doppelidentität auzuhalten, die ihnen erlaubte sich nach Bedarf nach der einen oder anderen Seite auszurichten. Andere wiederum scheiterteten, endeten auf der Straße. ${ }^{36}$

Die Konfrontation mit den aus Deutschland zurückgekehrten scharzen Deutschen veränderte die Wahrnehmung des Deutschen in Namibia. Verändert hat sich die Wahrnehmung aber auch im Zuge des Zustroms von Deutschen nach der Unabhängigkeit, wie sie etwa im Rahmen der deutschen Entwicklungszusammenarbeit nach Namibia kamen. Dabei geht es vor allem um die Deutschen, die ihre Arbeit in Namibia als Teil des bis zur Unabhängigkeit des Landes praktizierten Engagments gegen Apartheid und Rassismus definierten. Für die in Namibia ansässigen Deut-

35 Das Thema der sogenannten DDR-kids war lange Jahre gesuchtes Thema der deutschen Medien. Brigitte Schmidt-Lauber widmete im Buch Die verkehrte Hautfarbe den DDR-Kindern einen Abschnitt im Kapitel Grenzfälle und Grenzüberschreitungen. (B. Schmidt-Lauber, Die verkehrte Hautfarbe. Ethnizität deutscher Namibier als Alltagspraxis. Hamburg 1998, S. 401ff.)

36 Einer derer, die die Integration bisher nicht geschafft haben, lebte über einige Jahre in der Familie des Autors dieses Beitrages - er ist nach wie vor unser foster-son - und berichtete dem Autor vor kurzem in sicherem Deutsch, dass er jetzt das richtige Oshiwambo spreche, das er im Gefängnis gelernt habe. 
schen sind diese Deutsche Deutschländern, zu denen man Distanz bezog. Ansässig in diesem Land seit Generationen kennt man sein Land, man weiß, was für dieses Land richtig und was falsch ist und nun kommen diese Deutschländer und wissen alles besser. Haben die oder wir hier gelebt und überlebt? Hinzukam, dass man als Deutscher in Namibia natürlich auch ein sehr klare Wahrnehmung von dem zu haben glaubte, was deutsch ist. Man liest schließlich die deutsche namibische Zeitung, man liest importierte Presse, man schickt die Kinder in die deutsche Schule, man kauft deutsche Bücher in einer der deutschen Buchhandlungen in Windhoek oder Swakopmund, man besucht Verwandte und Bekannte in Deutschland und bekommt Besuch aus Deutschland. ${ }^{37}$ Deutschländer, die sich auch sprachlich vom alt Deutschnamibier, dem Südwester, unterscheiden, weil sie kein Südwester-deutsch sprechen, ${ }^{38}$ hatten es schwer Fuß zu fassen, sofern sie sich nicht dazu entschieden, die ansässige deutsche Gemeinde zu meiden und sich anderen Bereichen der namibischen Gesellschaft zuzuwenden. Annäherungen brauchten oft Jahre. Wahrnehmungsänderungen waren nötig, um hinter zu vordergründigen Politsierungen gegenseitiges Verständnis zu entwickeln. Geholfen hat dabei der zunehmende Abstand zur Zeit der Auseinandersetzungen, die diese Politisierunge produzierten. Politische Gegensätze aus der Vorunabhängigkeitszeit haben 17 Jahre nach der Unabhängigkeit an Bedeutung verloren. Wer zum Beispiel heute die Universität besucht, kennt Apartheid im Zweifel nur aus zweiter Hand. Geholfen hat aber auch afrikanische Wahrnehmung, die Widersprüche anders auszuhalten oder auch anders auszublenden vermag. Während es in Deutschland im Jahre 2006 offensichtliche politische Signalwirkung hate, die Münchener Von-Trotha-Straße im Andenken an die unter dem Befehl von Trotha Ermordeten in Herero-Straße umzubenennen, stehen in Namibia Symbole aus der deutschen Kolonialzeit unbefragt neben Symbole der Unabhängigkeit: Die nach dem Bremer Kaufmann Lüderitz, der den Weg zur Kolonie Deutsch Südwestafrika bereitete, benannte Lüderitz-Straße Windhoeks mündet in die Fidel-Castro-Straße! Dies wird auch vermutlich so bleiben, es sei denn, man benötigt eine Straße zur Bennung nach einer für das Land wichtigen Personlichkeit: Dann mag die Lüderitz-Straße verschwinden.

In Swakomund wurde vor nicht allzu langer Zeit aus der nach Kaiser Wilhelm benannten Straße eine zu Ehren des namibischen Gründungspräsidenten Sam Nujoma. Deutsche Geschäftsleute dieser gerne besuchten Küsten- und Wüstenstadt,

37 Im übrigen, so fügt der Autor dieses Beitrages im Sinne des Berichtes über Hunde und Deutsche ein, hat man mindestens zwei vielleicht gar drei Hunde, es gibt Tierkliniken, ein Tierwaschsalon, Geschäfte, die alle Arten von Hundefutter anbieten. Da im übrigen der Autor und seine Familie wie jeder gute Weißnamibier Hunde halten (es sind drei: Pinka, Bärchen und PataPata) war das Lesen der Arbeit von Ndonko für ihn nicht minder irritierend als die Herausgeber von Inspecting Germany.

38 Das Deutsch der Deutsch-Namibier hat einen Akzent, den man in Deutschland nicht findet, kennt leichte grammatikalische Abweichungen zum Standard-Hochdeutsch und benutzt zahlreiche Lehnwörter, zum größeren Teil aus dem Afrikaans, zum geringeren aus dem Englischen. 
die Kaisernostalgie als Bestantdteil der Attraktivität der Stadt sehen, protestierten gegen die Umbenennung und verloren im Stadtrat. Heute sieht man einige der ausgemusterten Kaiserstraßenschilder hinter den offiziellen als Hausdekoration angebracht. Auch das ist Deutsches im afrikanischen Visier!

\section{Zusammenfassung}

Wie sehen Afrikaner Deutsches? Sehen sie Deutsches unterschieden, von dem, was andere Weiße charaterisiert? Deutsches wird nur eingeschränkt wahrgenommen. Was die Wahrnehmung bestimmt, ist der koloniale oder postkoloiale Weiße, der eben auch Deutscher sein kann.

Dass Afrikaner Deutsches, oder allgemeiner Weißes ins Visier nehmen ist neu. Europa, die westliche Welt, war gewohnt, Afrika anzuschauen, aber nicht von Afrikanern angeschaut zu werden. Der verkehrten Ethnologie, wie sie Montesquieus Lettres Persannes dokumentieren, folgte eine weitere Verkehrung der Wahrnehmung durch Afrikaner. Sie nahmen Weißes (Deutsches) wahr und gestalteten das Wahrgenommene kreativ. Die Kultur der Colonschnitzerei ist ein Beispiel der Verarbeitung, in der wie auch beim heutigen Gedenken an den Völkermord an den Herero im damaligen Deutsch Südwestafrika Symbole deutscher Autorität und Übermacht zum Bestandteil der eigenen Kultur werden.

Die Hunde und die Deutschen wurde zu einem viel beachteten Beitrag eines afrikanischen Ethnologen auf einer internationalen Konferenz zum Thema Inspecting Germany. Deutsches in Namibia hat eine besondere Qualität durch die Präsenz deutscher Namibier, nach der Unabhängigkeit Zugezogener und schwarzer Namibier, die ihre Kindheit und Jugend in der DDR verbrachten.

\section{Summary}

How is the German seen by Africans? Does the African view distinguish between the German and the White? The German is only exceptionally seen as as distinct from other Whites. It is the colonial or postcolonial white (who may be German) that the African view is primarily concerned with.

The fact that Africans perceive what is German or more generally explore the attitudes and characteristcs of whites is novel. Europe, the western world was used to look at Africa, but not to be looked at by Africans. The upside-down anthropology, as it is documented in Montesquieu's Lettres Persannes was followed by another upside-down perception by Africans themselves. Africans perceived the white (the German) and transformed what they perceived in a creative manner. The culture of colon woodcarving is an example of this transformation. What the colon culture does is the transformation of symbols of white authority and power into African culture. This is also what happens when the Herero of today commemorate the genocide committed against them by Germany in 1904. 
Dogs and the Germans is the topic of a well-noted contribution by an African anthropologist to the international conference Inspecting Germany. German culture has a special quality in Namibia because of the presence of German Namibians, those who came to Namibia after the independence of the country, and black Namibian, who spent their childhood and youth in the GDR.

Manfred O. Hinz, The dismay being observed. Africans explore the German

\section{Deutsche Einheit auch beim Wähler?}

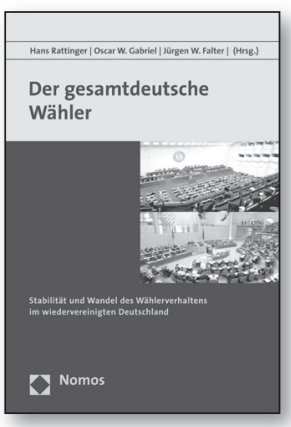

Der gesamtdeutsche Wähler

Stabilität und Wandel des Wählerverhaltens

im wiedervereinigten Deutschland

Herausgegeben von Hans Rattinger, Oscar W. Gabriel und Jürgen W. Falter 2007, 438 S., brosch., 49,- $€$, ISBN 978-3-8329-2545-1

Der Band widmet sich dem veränderten Wahlverhalten in Deutschland: Sind traditionelle gesellschaftliche Konflikte auch heute noch von Relevanz, oder treten neue Gegensätze u. a. zwischen Alt und Jung in den Vordergrund? Orientiert sich die Stimmabgabe zunehmend am politischen Spitzenpersonal? Hat die veränderte Bedeutung der Medien einen Einfluss auf das Wahlverhalten?

\section{Tipp:}

\section{Politische Integration im} vereinten Deutschland

Von Markus Linden 2006, 324 S., brosch., 39,-€, ISBN 978-3-8329-2206-1

Bitte bestellen Sie bei Ihrer Buchhandlung oder bei Nomos $607221 / 2104-37$ | www.nomos.de | sabine.horn@nomos.de 